\title{
Downregulation of microRNA-374a predicts poor prognosis in human glioma
}

\author{
QIANG DONG $^{1 *}$, GUOQIANG YUAN ${ }^{2 *}$, MIN LIU $^{3 *}$, QIQI XIE $^{4}, J_{A N H O N G ~ H U}{ }^{1}$, \\ MAOLIN WANG ${ }^{1}$, SHANGYU LIU $^{1}$, XIAOJUN MA ${ }^{1}$ and YAWEN PAN ${ }^{1,2}$ \\ ${ }^{1}$ Department of Neurosurgery, The Second Hospital of Lanzhou University; \\ ${ }^{2}$ Institute of Neurology, The Second Hospital of Lanzhou University, Lanzhou, Gansu 730030; \\ ${ }^{3}$ Department of Pharmacy, Hebei North University, Zhangjiakou, Hebei 075000; ${ }^{4}$ Department of Orthopaedics, \\ The Second Hospital of Lanzhou University, Lanzhou, Gansu 730030, P.R. China
}

Received July 8, 2018; Accepted December 3, 2018

DOI: 10.3892/etm.2019.7190

\begin{abstract}
Certain microRNAs (miRNAs/miRs) may be used as prognostic biomarkers in various types of cancer. The purpose of the present study was to identify miRNAs that were abnormally expressed in glioma of different grades, and to evaluate their clinical implications in patients with glioma. The differentially expressed miRNAs were evaluated from the expression profiles of six glioma tissues (three low-grade and three high-grade gliomas) determined using a microarray platform. Reverse transcription-quantitative polymerase chain reaction analysis was used to further verify the aberrant expression of the candidate miRNA in a set of 42 patients and 5 healthy controls. The miRNA target genes were predicted and the protein-protein interaction network was generated; furthermore, functional enrichment analysis of the target genes in Gene Ontology (GO) terms and Kyoto Encyclopedia of Genes and Genomes (KEGG) pathways was performed. Kaplan-Meier curves and Log-rank analysis, as well as multivariate Cox regression analysis were performed to assess the association of the candidate miRNA with patient survival. A total of 15 differentially expressed miRNAs, including 13 downregulated and 2 upregulated miRNAs, were identified by comparison of low-grade and high-grade glioma tissues. The miR-374a expression of high-grade gliomas was significantly lower than that of low-grade gliomas (fold change, $-4.43 ; \mathrm{P}=0.027$ ). The expression levels of miR-374a gradually decreased with the increase of the pathological grade of glioma. Pearson's Chi-square test was used to determine the association of miR-374a expression
\end{abstract}

Correspondence to: Professor Yawen Pan, Department of Neurosurgery, The Second Hospital of Lanzhou University, 82 Cuiyingmen Road, Chengguan, Lanzhou, Gansu 730030, P.R. China E-mail: panyw2018@163.com

*Contributed equally

Key words: glioma, prognosis, microarray, microRNA-374a with several clinicopathological factors. Furthermore, low expression of miR-374a was determined to be an independent prognostic marker and that it was significantly associated with overall survival $(\mathrm{P}=0.0213)$. $\mathrm{GO}$ and $\mathrm{KEGG}$ pathway analysis revealed that the target genes of miR-374a may be involved in the regulation of the RNA polymerase II promoter and mTOR signaling pathway. The four hub genes (CCND1, SP1, CDK4, $C D K 6$ ) were also identified by PPI network analysis. In conclusion, the present study indicated that miR-374a may be used as a promising prognostic biomarker for the screening of high-risk populations and for the assessment of the prognosis of patients with glioma.

\section{Introduction}

Glioma is the most common type of primary brain tumor in adults, accounting for $\sim 46 \%$ of intracranial tumors, with an incidence rate of $3-10 / 10$ million, corresponding to $1-3 \%$ of detected malignancies worldwide $(1,2)$. Grade-I and -II gliomas are classified as benign and low-grade gliomas, while grade-III and -IV gliomas are classified as high-grade gliomas, according to the World Health Organization (WHO) grading system (3). The current standard treatment for gliomas mainly includes surgery, followed by radiotherapy and chemotherapy. However, the therapeutic effect is not satisfactory. The median survival time of glioblastoma multiforme (GBM; grade 4 astrocytoma) patients remains poor, ranging from 12 to 15 months, 2 to 5 years and 6 to 8 years for grade IV, III and I-II gliomas, respectively according to the WHO grading system (4) Previous studies have proven that cytogenetic and molecular analyses have a vital role in predicting the remission and survival rates of patients. The mutation status of isocitrate dehydrogenase, NADP+, the heterozygous deletion at the chromosomal position $1 \mathrm{p} / 19 \mathrm{q}$ and the methylation of the O-6-methylguanine-DNA methyltransferase promoter (5-7) have been progressively used as diagnostic and prognostic markers for the comprehensive assessment of glioma patients. However, biological differences between individual patients are apparent and novel biomarkers are required to fully evaluate the prognosis of patients and predict the effectiveness of glioma treatment. 
MicroRNAs (miRNAs/miRs) are a class of short endogenous non-coding RNA molecules that are 18 to 25 nucleotides in length and exert a tumor-regulatory function at the post-transcriptional level by binding to the 3'-untranlated region (3'-UTR) and frequently to the 5'-UTR of mRNA molecules. Accumulating studies have identified multiple dysregulated miRNAs, which may serve as key molecules in cancer progression, and regulate various biological processes, including proliferation, differentiation, apoptosis and survival (8-10). Previous studies have demonstrated a significant difference in the expression profile of miRNAs between healthy subjects and patients with glioma, suggesting that the expression levels of certain miRNAs are associated with the overall survival (OS) of glioma patients (11-14). Therefore, the identification of differentially expressed miRNAs is of great importance in order to evaluate the early prognosis of glioma patients.

In the present study, a microarray-based analysis was performed to recognize differentially expressed miRNAs in gliomas by comparing miRNA expression profiles among low-grade and high-grade gliomas. Furthermore, the differential expression of miR-374a in human glioma tissues was confirmed by reverse transcription-quantitative polymerase chain reaction (RT-qPCR) analysis. The prognostic value of this differentially expressed miRNA was then investigated. Furthermore, a bioinformatics analysis based on miRNA target gene databases was used to identify target genes of miR-374a. The GO and KEGG analysis were performed and the hub genes were analyzed by construction of a protein-protein interaction (PPI) network for the target genes. The aim of the present study was to obtain novel prognostic and predictive biomarkers for glioma, and to investigate the potential mechanisms of glioma progression.

\section{Patients and methods}

Patients and tissue specimens. Glioma tissues (15 grade II, 13 grade III and 20 grade IV) from a total of 48 patients were collected from the Department of Neurosurgery of the Second Hospital of the Lanzhou University (Gansu, China), from January 2013 to December 2016. The 5 normal brain tissues from patients without glioma who underwent surgery for other reasons, including cerebral trauma. Following surgical removal, the tissue samples were snap-frozen in liquid nitrogen and stored at $-80^{\circ} \mathrm{C}$ until used for RNA isolation. Patients who had received chemotherapy or radiotherapy prior to surgery were excluded. During the follow-up, OS was observed from the date of diagnosis to the date of patient death and/or the last census date in case of the patient being alive.

Microarray. A total of six glioma tissues (3 low-grade and 3 high-grade gliomas) were next analyzed using microarray methodologies in order to screen for the expression of miRNAs. A total of $1 \mu \mathrm{g}$ of total RNA was extracted from each tumor sample using the TRIzol reagent (Invitrogen; Thermo Fisher Scientific, Inc., Waltham, MA, USA), and first-strand complementary (c)DNA synthesis was performed using the miRNA First-Strand cDNA Synthesis kit (cat. no. AS-MR-004; Arraystar, Rockville, MD, USA) following the manufacturer's protocols. The miRStar ${ }^{\mathrm{TM}}$ Human Cancer Focus miRNA
PCR Array (cat. no. AS-MR-0033; Arraystar) was applied on the ABI PRISM7900 system (Applied Biosystems; Thermo Fisher Scientific, Inc.). Each 384-well miRStar ${ }^{\mathrm{TM}}$ Human Cancer Focus miRNA PCR Array contained 184 miRNAs linked to human cancers, nine wells for different housekeeping miRNAs, a genomic DNA contamination control, three replicate RT controls and three replicate positive PCR controls. The values of the quantification cycle $(\mathrm{Cq})$ that were obtained for quantification were used for the calculation of fold changes in miRNA abundance according to the $2^{-\Delta \Delta C q}$ method (15). The raw data were processed by the following workflow: Background detection, RMA global background correlation, quantile normalization, median polish adjustment was performed and $\log 2$-transformation with miRNA QC tool software (Affymetrix; Thermo Fisher Scientific, Inc.) (16).

$R T-q P C R$. Total RNA was extracted from tissues using TRIzol reagent (Invitrogen; Thermo Fisher Scientific, Inc.) according to the manufacturer's protocols. cDNA was randomly synthesized from $2 \mu \mathrm{g}$ total RNA using the miRcute Plus miRNA First-Strand cDNA Synthesis Kit (cat. no. KR211; Tiangen Biochemical Technology Beijing Co. Ltd., Beijing, China) according to the manufacturer's instructions. RT products were amplified using SYBR Green PCR (cat. no. FP411; Tiangen Biochemical Technology Beijing Co. Ltd.) on a Bio-Rad CFX96 real-time PCR system (Bio-Rad Laboratories, Hercules, CA, USA). The reaction was performed according to the manufacturer's instructions. The relative mRNA expression data were acquired and analyzed using the $2^{-\Delta \Delta \mathrm{Cq}}$ method, and the expression values were normalized to U6, which was used as an internal control. The primers used in the present study were as follows: miR-374a forward, 5'-CGGCGGTTATAA TACAACCTG-3' and reverse, 5'-AGCTCGAGTGGAAGT CTGTGCA-3'; U6 forward, 5'-CTCGCTTCGGCAGCACAT ATA-3' and reverse, 5'-AACGCTTCACGAATTTGCGT-3'.

Data analysis. The median value of the expression levels of miR-374a was used as the cut-off point, and the patients were divided into a high-level and a low-level group. The expression levels of miR-374a between glioma and normal brain tissues were compared with the independent-samples t-test. Pearson's Chi-square test was used to analyze the association between miR-374a expression levels and the clinicopathological characteristics of the patients. The OS rates of the patients in the high-level and low-level groups were evaluated using the Kaplan-Meier method. Furthermore, univariate and multivariate Cox proportional hazard regression models were used to evaluate the prognostic value of multiple variables, including miR-374a expression, sex, age and Karnofsky performance status (KPS) score.

Bioinformatics analysis. The target genes of miR-374a were predicted using TargetScan (http://www.targetscan. org/) (17), miRTarBase (http://mirtarbase.mbc.nctu.edu. tw/php/search. php) (18), starBase (http://starbase.sysu. edu.cn/browseIntersectTargetSite.php) (19) and miRDB (http://www. mirdb.org/) (20). To enhance the reliability of the bioinformatics analysis, the overlapping target genes were identified using a Venn diagram. To further investigate the functions of these consensus target genes, the Database for 
A

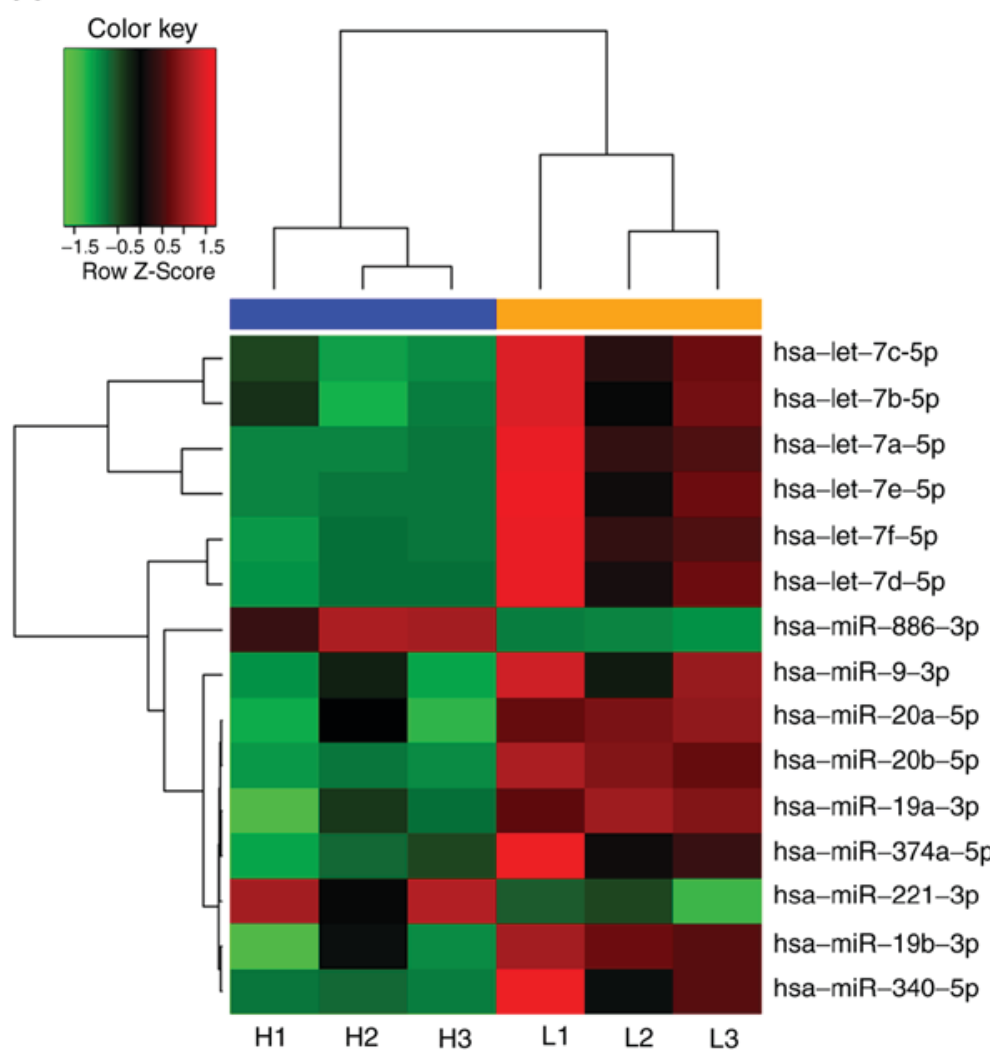

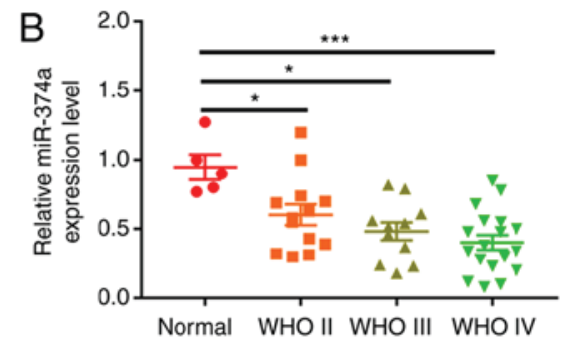

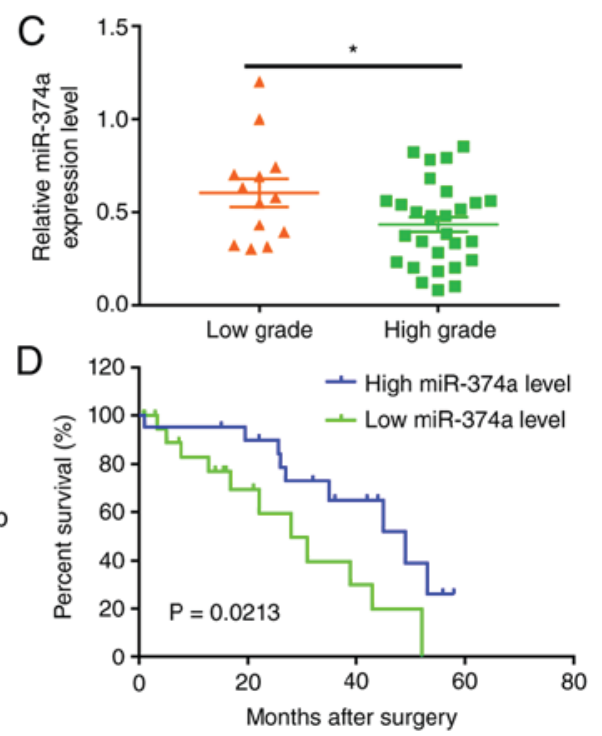

Figure 1. (A) Heatmap of the differentially expressed miRNAs between low-grade gliomas ( $\mathrm{n}=3$ ) and high-grade gliomas ( $\mathrm{n}=3$ ). Each row represents the expression levels of a specific miRNA and each column represents a single sample: Orange color denotes low-grade gliomas, whereas blue denotes high-grade gliomas. (B) The relative expression levels of miR-374a in glioma samples of different grades (n=42) were significantly decreased compared with those in normal tissues $(n=5)$; furthermore, the expression of miR-374a slightly increased in glioma with increasing grade. (C) miR-374a expression levels in high-grade glioma tissues $(\mathrm{n}=13)$ and low-grade glioma tissues $(\mathrm{n}=29 ; \mathrm{P}<0.05) .{ }^{*} \mathrm{P}<0.05,{ }^{* * *} \mathrm{P}<0.001$. (D) Kaplan-Meier curves for overall survival in patients with gliomas stratified according to high/low expression of miR-374a. miRNA/miR, microRNA; hsa, Homo sapiens; H, high-grade gliomas; L, low-grade gliomas; WHO, World Health Organization.

Annotation, Visualization and Integrated Discovery (DAVID) bioinformatics tool (https://david.ncifcrf.gov/) and mirPath v.3 (http://snf-515788.vm.okeanos.grnet.gr/) were used to perform gene ontology (GO) and Kyoto Encyclopedia of genes and genomes (KEGG) pathway enrichment analyses. These analyses aimed to predict protein interactions, which included physical and functional associations. The present study used Search Tool for the Retrieval of Interacting Genes (STRING; https://string-db.org/) to construct the PPI network for target genes (minimum required interaction score. $>0.4$ ). In addition, Cytoscape software version 3.6.0 (http://www.cytoscape. org/download-platforms.html) was used for visualization of the PPI networks.

Statistical analysis. All analyses were performed using SPSS 17.0 software (SPSS, Inc., Chicago, IL, USA) and GraphPad Prism 5 software (GraphPad Software Inc., La Jolla, CA, USA). Data calculations from each experiment were performed independently at least three times and values are expressed as the mean \pm standard error of the mean. Differences between groups were assessed by one-way analysis of variance followed by an LSD post-hoc test and a Student's t-test. The Kaplan-Meier method was used to estimate the OS curves. $\mathrm{P}<0.05$ was considered to indicate statistical significance.

\section{Results}

miRNA profiling. The miRNA profile was analyzed for low-grade $(n=3)$ and high-grade gliomas $(n=3)$ using the miRStar ${ }^{\mathrm{TM}}$ Human Cancer Focus miRNA PCR Array, which allowed for the assessment of the expression levels of 184 miRNAs associated with cancer. The median expression levels of each miRNA in each of the two groups were calculated, and the differences between them were determined using the t-test. $\mathrm{P}<0.05$ was considered to indicate significant differences in expression and a fold change of $>2$ was set as the cut-off value. A total of 15 miRNAs, including 13 downregulated and 2 upregulated miRNAs, were identified between low-grade and high-grade glioma tissues(Fig. 1A; Table I). As the differences in multiples of miR-374a were the largest, miR-374a was selected for further study. It was revealed that the expression levels of miR-374a in the high-grade glioma tissues were lower than those in the low-grade glioma tissues $(\mathrm{P}=0.027)$.

miR-374a is downregulated in glioma tissues. To validate the results that were obtained by the microarray analysis, the expression levels of miRNA-374a we evaluated in glioma $(n=42)$ and normal brain tissues $(n=5)$ by RT-qPCR. Significantly 
lower miR-374a expression levels were observed in glioma tissues compared with those in the adjacent normal tissues and the expression of miR-374a decreased with increasing glioma grade $(\mathrm{P}<0.05$; Fig. 1B and $\mathrm{C})$. These RT-qPCR data confirmed that the results of the microarray analysis were reliable.

Association of miR-374a expression levels with clinicopathological characteristics and OS of glioma patients. The association of the miR-374a expression levels with the demographic and clinicopathological characteristics of the glioma patients was then assessed (Table II). The median expression levels of miR-374a were used as a cut-off value, and all patients were divided into a high-level group $(n=21)$ and a low-level group $(n=21)$. The expression levels of miR-374a were associated with the KPS score $(\mathrm{P}=0.032)$ and the WHO grade $(\mathrm{P}=0.028$; Table II). However, the miR-374a expression levels were not significantly associated with any of the other parameters assessed, including age and sex ( $\mathrm{P}>0.05)$.

Kaplan-Meier curves and the log-rank test were used in order to evaluate the prognostic value of miR-374a in glioma. The results indicated that patients with low expression levels of miR-374a had a significantly shorter OS than those with high miR-374a expression levels $(\mathrm{P}=0.021$; Fig. 1D). Furthermore, Cox regression analysis indicated that the expression levels of miR-374a were associated with the OS of glioma patients (hazard ratio, 0.472 ; $95 \%$ confidence interval, $0.125-1.733 ; \mathrm{P}<0.05)$ and the WHO grade was also significantly associated with the OS of glioma patients (hazard ratio, 1.914; 95\% confidence interval, 1.362-3.885; $\mathrm{P}<0.05$; Table III) Collectively, these data suggest that miR-374a may be a prognostic factor regarding OS in patients with glioma.

Target prediction and functional analysis. To elucidate the potential biological function of miR-374a in glioma, the target genes of miR-374a were predicted using the TargetScan, miRTarBase, StarBase and miRDB online analysis tools. A total of 35 overlapping genes among the four different tools were identified (Fig. 2). Subsequently, a functional enrichment analysis was performed to investigate the biological functions of these consensus target genes. The enriched GO terms in the categories biological process (BP), cellular component and molecular function were identified. In the category $\mathrm{BP}$, the target genes were mostly involved in metabolic processes (Fig. 3).

KEGG pathway analysis and PPI network. The KEGG pathways of the miR-374a target genes were predicted using DIANA-miRPath in order to identify miRNA-mRNA regulatory signaling pathways in glioma (21-23). The most significant biological pathways were associated with tumor progression from glial progenitor cells to primary GBM, including calcium metabolism, ErbB, mammalian target of rapamycin (mTOR) and several cell cycle-associated pathways (Fig. 4A). PPI network analysis of all target genes using the STRING database revealed 125 interactions involved. The nodes with a degree of interaction of $\geq 7$ were defined as hub genes, including cyclin D (CCND)1, specificity protein 1 (Sp1), cyclin-dependent kinase (CDK)4 and CDK6 (Fig. 4B).
Table I. Differential expression miRNAs between high-grade and low-grade glioma groups.

\begin{tabular}{lcc}
\hline miRNA ID & $\log 2$ fold change & P-value \\
\hline hsa-let-7a-5p & -3.10 & 0.010 \\
hsa-let-7b-5p & -2.28 & 0.032 \\
hsa-let-7c-5p & -2.23 & 0.012 \\
hsa-let-7d-5p & -2.82 & 0.018 \\
hsa-let-7e-5p & -3.46 & 0.022 \\
hsa-let-7f-5p & -3.29 & 0.011 \\
hsa-miR-19a-3p & -2.60 & 0.008 \\
hsa-miR-19b-3p & -3.02 & 0.021 \\
hsa-miR-20a-5p & -2.31 & 0.017 \\
hsa-miR-221-3p & 2.58 & 0.026 \\
hsa-miR-340-5p & -4.15 & 0.044 \\
hsa-miR-374a-5p & -4.43 & 0.027 \\
hsa-miR-886-3p & 3.82 & 0.001 \\
hsa-miR-9-3p & -3.79 & 0.049
\end{tabular}

miRNA/miR, microRNA; hsa, Homo sapiens.

\section{Discussion}

miRNAs are regulators of post-transcriptional gene expression and are involved in multiple complex pathways (24). A large number of studies have reported that certain miRNA are involved in the regulation of tumor progression, and may have tumor-promoting or tumor-suppressive roles with regard (25-27). miRNAs may be used as prognostic indicators and therapeutic targets in gliomas $(28,29)$. In the present study, comprehensive miRNA profiling of high-grade vs. low-grade gliomas was performed by microarray analysis. A total of 15 significantly altered miRNAs in high-grade vs. low-grade gliomas were identified. In addition, a novel miRNA, miR-374a, was identified to be differentially expressed between normal and glioma tissues, which has not been previously reported. Therefore, miR-374a was selected for subsequent investigation. It was demonstrated that miR-374a was significantly lower expressed in high-grade vs. low-grade gliomas. The miRNA expression was verified by RT-qPCR in order to confirm the results of the microarray profiling. Furthermore, the present results indicated that miR-374a may be considered a potential prognostic biomarker in glioma due to the association of its expression levels with the long-term survival of glioma patients.

A number of studies have also reported that the abnormal expression of miR-374a are involved in multiple types of cancer. Li et al (30) revealed that deregulation of miR-374a may be involved in the development and regulation of cisplatin resistance in ovarian cancer cells. Wu et al (31) demonstrated that the expression levels of miR-374a were significantly lower in lung adenocarcinoma compared with those in the adjacent normal tissues. Furthermore, regulation of transforming growth factor $\alpha$ gene expression by miR-374a inhibited the proliferation, migration and invasion of lung adenocarcinoma cells (31). Slattery et al (32) demonstrated that the expression levels of miR-374a were downregulated in colorectal cancer, 
Table II. Association of the expression level of miR-374a with clinicopathological factors of glioma.

miR-374a expression (n)

\begin{tabular}{|c|c|c|c|c|}
\hline \multirow[b]{2}{*}{ Clinicopathological feature } & \multirow[b]{2}{*}{ Patients (n) } & & \multirow[b]{2}{*}{ P-value } \\
\hline & & Low & High & \\
\hline Age (years) & & & & 0.334 \\
\hline$\geq 50$ & 15 & 6 & 19 & \\
\hline$<50$ & 27 & 15 & 12 & \\
\hline Sex & & & & 0.533 \\
\hline Male & 24 & 11 & 13 & \\
\hline Female & 18 & 10 & 8 & \\
\hline WHO grade & & & & 0.028 \\
\hline II & 13 & 5 & 8 & \\
\hline III/IV & 29 & 16 & 13 & \\
\hline KPS score & & & & 0.032 \\
\hline$\geq 90$ & 11 & 2 & 9 & \\
\hline$<90$ & 31 & 9 & 12 & \\
\hline
\end{tabular}

KPS, Karnofsky performance status; WHO, World Health Organization; miR, microRNA.

Table III. Univariate and multivariate analyses of prognostic factors in patients with glioma.

\begin{tabular}{|c|c|c|c|c|c|c|}
\hline \multirow[b]{2}{*}{ Variable } & \multicolumn{3}{|c|}{ Univariate analysis } & \multicolumn{3}{|c|}{ Multivariate analysis } \\
\hline & HR & $95 \% \mathrm{CI}$ & P-value & HR & $95 \% \mathrm{CI}$ & P-value \\
\hline Age $(\geq 50$ vs. $<50$ years $)$ & 1.414 & $0.591-3.384$ & 0.436 & 1.031 & $0.421-2.524$ & 0.946 \\
\hline Sex (male vs. female) & 1.19 & $0.497-2.851$ & 0.696 & 1.155 & $0.444-3.007$ & 0.768 \\
\hline KPS ( $\geq 90$ vs. $<90)$ & 0.274 & $0.089-0.844$ & 0.024 & 1.025 & $0.390-2.699$ & 0.056 \\
\hline WHO grade (II vs. III/IV) & 1.51 & $1.163-2.98$ & 0.027 & 1.914 & $1.362-3.885$ & 0.036 \\
\hline miR-374a expression (low vs. high) & 0.379 & $0.154-0.936$ & 0.021 & 0.472 & $0.125-1.733$ & 0.016 \\
\hline
\end{tabular}

KPS, Karnofsky performance status; WHO, World Health Organization; miR, microRNA; HR, hazard ratio; CI, confidence interval.

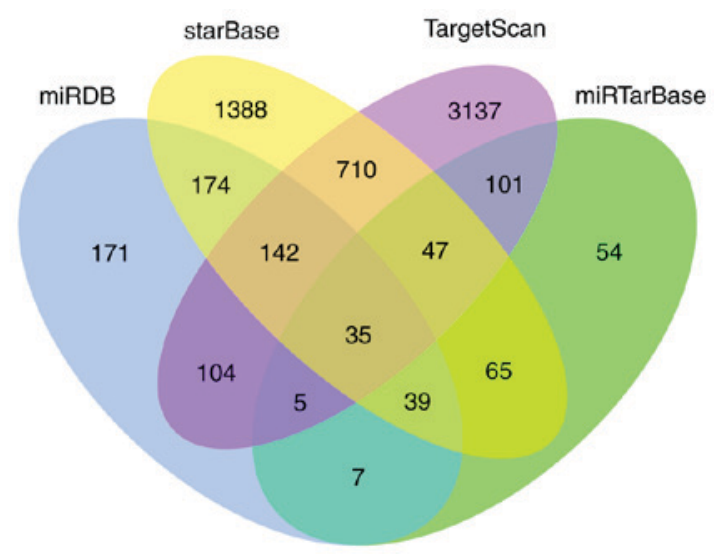

Figure 2. Venn diagram of overlapping target genes predicted using different online analysis tools (TargetScan, miRDB, starbase and miRTarBase).

whereas low miR-203 expression levels were associated with worse clinicopathological data and shorter OS time. However, $\mathrm{Xu}$ et al (33) reported that miR-374a acts as a tumor promoter in gastric cancer, where it and promotes cell proliferation, migration and invasion via the regulation of SRC kinase signaling inhibitor 1 expression levels. In addition, Pan et al (34) highlighted that the expression levels of miR-374 were decreased in glioma tissues and were associated with the prognosis of glioma patients, which is consistent with the results of the present study.

It has been reported that the aberrant expression of certain miRNAs is associated with the development of cancer via the abnormal regulation of multiple BPs and signaling pathways (35). To further elucidate the molecular function of miR-374a and its target genes, functional enrichment analyses of the target genes in GO terms and KEGG pathways were performed. The GO analysis demonstrated that the terms in the category BP included the regulation of the RNA transcription and the nucleic acid metabolic processes. Furthermore, several of the enriched pathways were associated with tumorigenesis, including ErbB, mTOR and cell cycle signaling pathways. The ErbB pathway is associated with tumor progression in the majority of cancer types, including glioma $(36,37)$. Furthermore, 


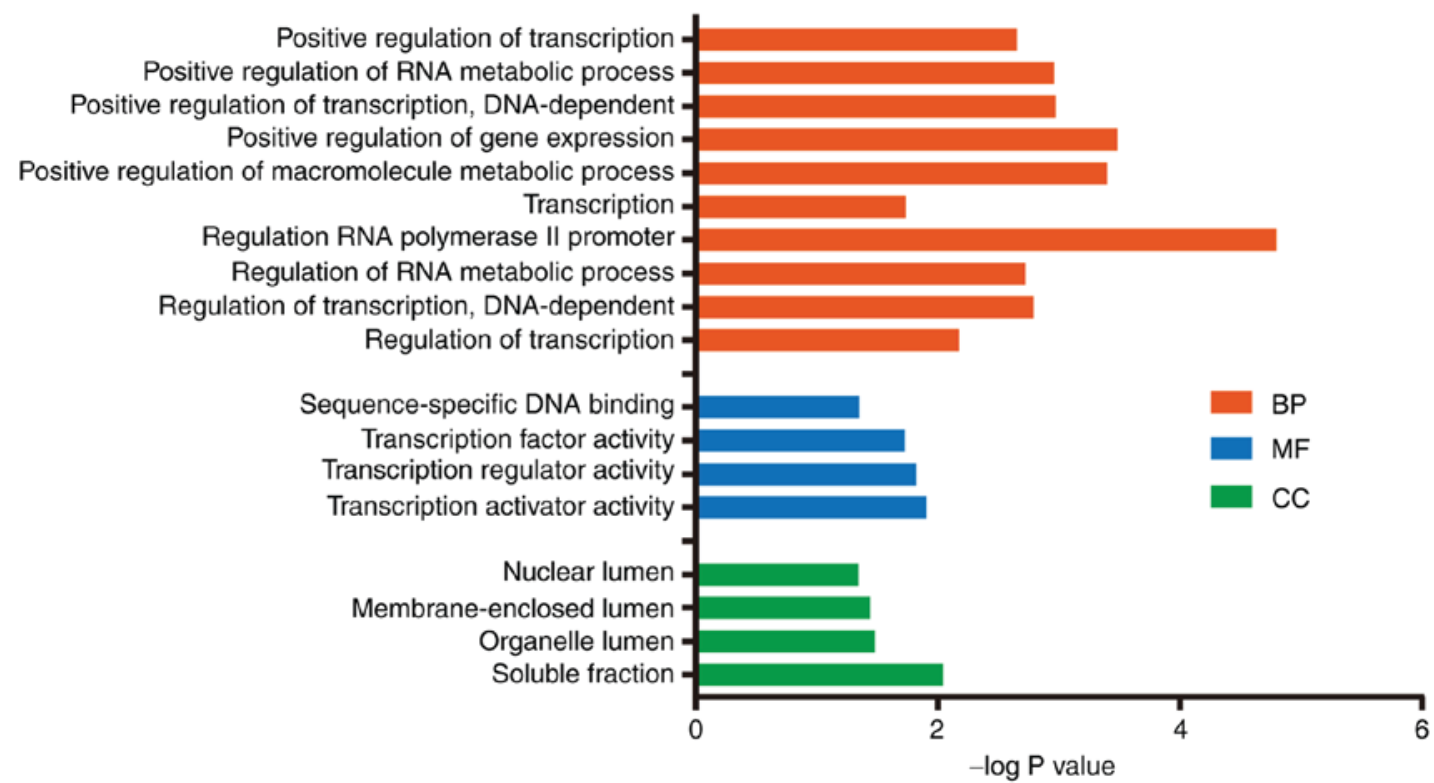

Figure 3. Significantly enriched Gene Ontology terms in the categories BP, MF and CC of the 35 consensus target genes. BP, biological process; MF, molecular function; CC, cellular component.

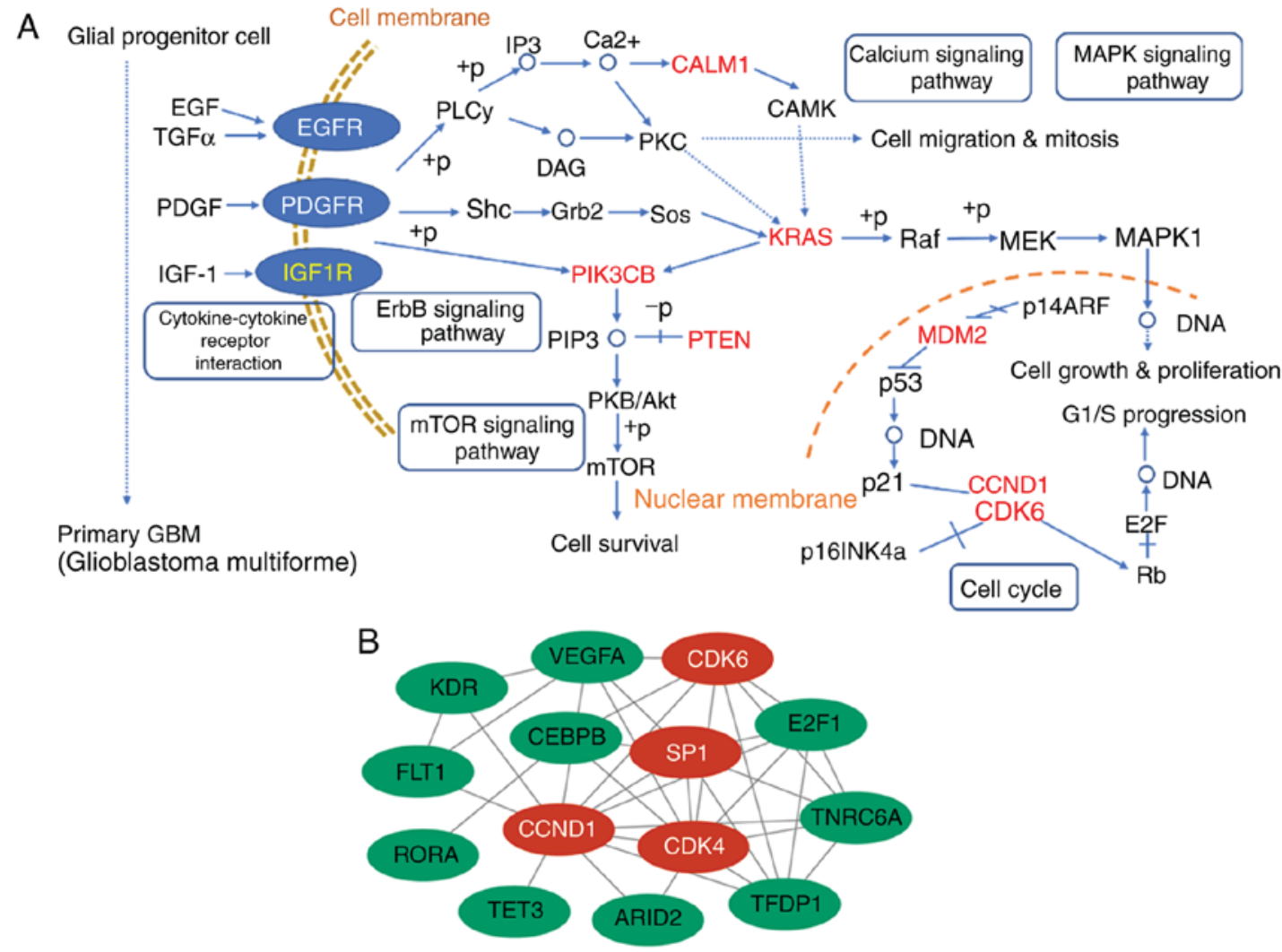

Figure 4. (A) KEGG pathway analysis based on predicted targets of miR-374a. These targets were identified in the Glioma Pathway using the DIANA-mirPath tool. The red color denotes the target gene of miR-374a. (B) Protein-protein interaction network constructed from target genes; red circles indicate hub genes. KEGG, Kyoto Encyclopedia of Genes and Genomes; miRNA/miR, microRNA; GBM, glioblastoma multiforme.

it was reported that the mTOR pathway is a crucial signaling pathway involved in the development of glioma (38).

In addition, four hub genes, CCND1, SP1, CDK6 and CDK4, were identified from the PPI of predicted target genes of miR-374a, which may be directly or indirectly involved in the development of glioma. CCND1 is a protein required for the progression from G1 phase to the S phase of the cell cycle. CCND1 overexpression is associated with early cancer onset (39) and tumor progression and decreased Fas expression, leading to increased chemotherapeutic resistance and protection from apoptosis (40). CDK4 and CDK6 are two members of the CDK family that bind to CCND1. A dysregulation of 
CDK4/6 may promote GBM proliferation; however, CDK4/6 kinase inhibitors were demonstrated to inhibit cell proliferation in subcutaneous glioma models (41). Sp1 belongs to the $\mathrm{Sp} /$ Kruppel-like factor family of transcription factors and is widely expressed in gliomas. $\mathrm{Sp} 1$ has an important role in the activation of oncogenes required for tumor survival (42). Guan et al (43) demonstrated that SP1 is upregulated in human glioma and may serve as a prognostic marker.

The primary limitation of the present study is that no luciferase reporter assays of the hub genes (CCND1, SP1, CDK6 and CDK4) were performed to validate the target genes of miR-374a. Therefore, further analyses are required to determine the mechanisms in the processes of malignant progression in gliomas. Future studies will use PCR to verify the expression levels of miR-374a in glioma cells and a combination of other molecular markers to successfully identify patients with OS.

In conclusion, the present study demonstrated that the downregulation of miR-374a is associated with reduced survival in glioma patients. miR-374a may be a potential prognostic factor for patients with glioma. However, the results of the present study should be verified using a larger sample size and further experimental research is required to confirm the functions of miR-374a in the progression of glioma.

\section{Acknowledgements}

Not applicable.

\section{Funding}

This project was supported by the Natural Science Foundation of Gansu (grant nos. 18JR3RA365 and 18JR3RA309), the Research Fund from the Project of the Healthy and Family Planning Commission of Gansu (grant no. GSWSKY-2014-31/2015-58), the Lanzhou Science and Technology Bureau Project (grant no. 2018-1-109) and the doctoral research fund and the Cuiying Science and Technology fund of Lanzhou University Second Hospital (grant nos. ynbskyjj2015-1-02/2015-2-11/2015-2-5 and CY2017-MS12/-MS15/CYXZ-01).

\section{Availability of data and materials}

The datasets used and/or analyzed during the present study are available from the corresponding author on reasonable request.

\section{Authors' contributions}

YP and QD initiated the project. QD, ML, GY, MW and JH performed the experiments. QD, ML, QX and GY analyzed the data. QX, JH, SL and XM generated the figures. YP, QD, GY and ML wrote the manuscript. All co-authors read and approved the final manuscript.

\section{Ethics approval and consent to participate}

The present study was approved by the Ethical Committee of the Second Hospital of the Lanzhou University. Written informed consent was provided by each patient or their guardians prior his/her participation in the study.

\section{Patient consent for publication}

Not applicable.

\section{Competing interests}

The authors declare that they have no competing interests.

\section{References}

1. Ohgaki $\mathrm{H}$ and Kleihues P: Epidemiology and etiology of gliomas. Acta Neuropathol 109: 93-108, 2005.

2. Schwartzbaum JA, Fisher JL, Aldape KD and Wrensch M: Epidemiology and molecular pathology of glioma. Nat Clin Pract Neurol 2: 494-503, 2006.

3. Louis DN, Perry A, Reifenberger G, von Deimling A, Figarella-Branger D, Cavenee WK, Ohgaki H, Wiestler OD, Kleihues P and Ellison DW: The 2016 World Health Organization classification of tumors of the central nervous system: A summary. Acta Neuropathologica 131: 803-820, 2016.

4. Stupp R, Hegi ME, Mason WP, van den Bent MJ, Taphoorn MJ, Janzer RC, Ludwin SK, Allgeier A, Fisher B, Belanger K, et al: Effects of radiotherapy with concomitant and adjuvant temozolomide versus radiotherapy alone on survival in glioblastoma in a randomised phase III study: 5-year analysis of the EORTC-NCIC trial. Lancet Oncol 10: 459-466, 2009.

5. Delgado-López PD and Corrales-García EM: Survival in glioblastoma: A review on the impact of treatment modalities. Clin Transl Oncol 18: 1062-1071, 2016.

6. Kleihues P, Burger PC, Aldape KD, et al: In: Louis DN, Ohgaki H, Wiestler OD, Cavenee WK, (eds). WHO classification of tumours of the central nervous system, 2007.

7. Abrunhosa-Branquinho AN, Bar-Deroma R, Collette S, Clementel E, Liu Y, Hurkmans CW, Feuvret L, Van Beek K, van den Bent M,Baumert BG and Weber DC: Radiotherapy quality assurance for the RTOG 0834/EORTC 26053-22054/NCIC CTG CEC.1/CATNON intergroup trial "concurrent and adjuvant temozolomide chemotherapy in newly diagnosed non-1p/19q deleted anaplastic glioma': Individual case review analysis. Radiother Oncol 127: 292-298, 2018.

8. Zhou L, Liu F, Wang X and Ouyang G: The roles of microRNAs in the regulation of tumor metastasis. Cell Biosci 5: 32, 2015.

9. Aakula A, Kohonen P, Leivonen SK, Mäkelä R, Hintsanen P, Mpindi JP, Martens-Uzunova E, Aittokallio T, Jenster G, Perälä M, et al: Systematic identification of MicroRNAs that impact on proliferation of prostate cancer cells and display changed expression in tumor tissue. Eur Urol 69: 1120-1128, 2016.

10. Zhu QN, Renaud H and Guo Y: Bioinformatics-based identification of miR-542-5p as a predictive biomarker in breast cancer therapy. Hereditas 155: 17, 2018.

11. Man HB, Bi WP and Man HH: Decreased microRNA-198 expression and its prognostic significance in human glioma. Genet Mol Res: 15, 2016.

12. Zhang J, Lv J, Zhang F, Che H, Liao Q, Huang W, Li S and Li Y: MicroRNA-211 expression is down-regulated and associated with poor prognosis in human glioma. J Neurooncol 133: 553-559, 2017.

13. Yuan GQ, Wei NL, Mu LY, Wang XQ, Zhang YN, Zhou WN and Pan YW: A 4-miRNAs signature predicts survival in glioblastoma multiforme patients. Cancer Biomark 20: 443-452, 2017.

14. Zhou MH, Zhou HW, Liu M and Sun JZ: The role of miR-92b in cholangiocarcinoma patients. Int J Biol Markers 33: 293-300, 2018.

15. Livak KJ and Schmittgen TD: Analysis of relative gene expression data using real-time quantitative PCR and the 2(-Delta Delta C(T)) method. Methods 25: 402-408, 2001.

16. Sarrion I, Milian L, Juan G, Ramon M, Furest I, Carda C, Cortijo Gimeno J and Mata Roig M: Role of circulating miRNAs as biomarkers in idiopathic pulmonary arterial hypertension: Possible relevance of miR-23a. Oxid Med Cell Longev 2015: 792846, 2015.

17. Ekimler S and Sahin K. Computational Methods for MicroRNA Target Prediction[J]. Genes 5 (3): 671-683, 2014. 
18. Hsu SD, Lin FM, Wu WY, Liang C, Huang WC, Chan WL, Tsai WT, Chen GZ, Lee CJ, Chiu CM, et al: miRTarBase: A database curates experimentally validated microRNA-target interactions. Nucleic Acids Res 39 (Database Issue): D163-D169, 2011.

19. Li JH, Liu S, Zhou H, Qu LH and Yang JH: starBase v2.0: Decoding miRNA-ceRNA, miRNA-ncRNA and protein-RNA interaction networks from large-scale CLIP-Seq data. Nucleic Acids Res 42 (Database Issue): D92-D97, 2014.

20. Vlachos IS, Kostoulas N, Vergoulis T, Georgakilas G, Reczko M, Maragkakis M, Paraskevopoulou MD, Prionidis K, Dalamagas T and Hatzigeorgiou AG: DIANA miRPath v.2.0: Investigating the combinatorial effect of microRNAs in pathways. Nucleic Acids Res 40: W498-W504, 2012.

21. Wong N and Wang X: miRDB: An online resource for microRNA target prediction and functional annotations. Nucleic eAcids Rse 43 (Database Issue): D146-D152, 2015.

22. Papadopoulos GL, Alexiou P, Maragkakis M, Reczko M and Hatzigeorgiou AG: DIANA-mirPath: Integrating human and mouse microRNAs in pathways. Bioinformatics 25: 1991-1993, 2009.

23. Fazi B, Felsani A, Grassi L, Moles A, D Andrea D, Toschi N, Sicari D, De Bonis P, Anile C, Guerrisi MG, et al: The transcriptome and miRNome profiling of glioblastoma tissues and peritumoral regions highlights molecular pathways shared by tumors and surrounding areas and reveals differences between short-term and long-term survivors. Oncotarget 6: 22526-22552, 2015.

24. Dai J, Li Q, Bing Z, Zhang Y, Niu L, Yin H, Yuan G and Pan Y: Comprehensive analysis of a microRNA expression profile in pediatric medulloblastoma. Mol Med Rep 15: 4109-4115, 2017.

25. Nohata N, Hanazawa T, Kinoshita T, Okamoto Y and Seki N: MicroRNAs function as tumor suppressors or oncogenes: Aberrant expression of microRNAs in head and neck squamous cell carcinoma. Auris Nasus Larynx 40: 143-149, 2013.

26. Babashah S and Soleimani M: The oncogenic and tumour suppressive roles of microRNAs in cancer and apoptosis. Eur J Cancer 47: 1127-1137, 2011.

27. Chen Y, Fu LL, Wen X, Liu B, Huang J, Wang JH and Wei YQ Oncogenic and tumor suppressive roles of microRNAs in apoptosis and autophagy. Apoptosis 19: 1177-1189, 2014

28. Xue L, Wang Y, Yue S and Zhang J: The expression of miRNA-221 and miRNA-222 in gliomas patients and their prognosis. Neurol Sci 38: 67-73, 2017.

29. Ji Y, Wei Y, Wang J, Gong K, Zhang Y and Zuo H: Correlation of microRNA-10b upregulation and poor prognosis in human gliomas. Tumour Biol 36: 6249-6254, 2015.

30. Li N, Yang L, Wang H, Yi T, Jia X, Chen C and Xu P: MiR-130a and MiR-374a function as novel regulators of cisplatin resistance in human ovarian cancer A2780 cells. PLoS One 10: e0128886, 2015.
31. Wu H, Liu Y, Shu XO and Cai Q: MiR-374a suppresses lung adenocarcinoma cell proliferation and invasion by targeting TGFA gene expression. Carcinogenesis 37: 567-575, 2016.

32. Slattery ML, Pellatt AJ, Lee FY, Herrick JS, Samowitz WS Stevens JR, Wolff RK and Mullany LE: Infrequently expressed miRNAs influence survival after diagnosis with colorectal cancer. Oncotarget 8: 83845-83859, 2017.

33. Xu X, Wang W, Su N, Zhu X, Yao J, Gao W, Hu Z and Sun Y: miR-374a promotes cell proliferation, migration and invasion by targeting SRCIN1 in gastric cancer. FEBS Lett 589: 407-413, 2015.

34. Pan Z, Shi Z, Wei H, Sun F, Song J, Huang Y, Liu T and Mao Y: Magnetofection based on superparamagnetic iron oxide nanoparticles weakens glioma stem cell proliferation and invasion by mediating high expression of MicroRNA-374a. J Cancer 7: 1487-1496, 2016.

35. Zhang C, Zhang C, Ma M and Dai D: Three-microRNA signature identified by bioinformatics analysis predicts prognosis of gastric cancer patients. World J Gastroenterol 24: 1206-1215, 2018.

36. Späth F, Andersson U, Dahlin AM, Langseth H, Hovig E, Johannesen TB, Grankvist K, Björkblom B, Wibom C and Melin B: Pre-diagnostic serum levels of EGFR and ErbB2 and genetic glioma risk variants: A nested case-control study. Tumour Biol 37: 11065-11072, 2016

37. Liu R, Qu Y, Chen L, Pu J, Ma S, Zhang X, Yang Q, Shi B, Hou P and Ji M: Genomic copy number gains of ErbB family members predict poor clinical outcomes in glioma patients. Oncotarget 8: 92275-92288, 2017.

38. Wang N, Zhang Q, Luo L, Ning B and Fang Y: $\beta$-asarone inhibited cell growth and promoted autophagy via $\mathrm{P} 53 / \mathrm{Bcl}-2 / \mathrm{Bclin}-1$ and P53/AMPK/mTOR pathways in Human Glioma U251 cells. J Cell Physiol 233: 2434, 2018.

39. Diehl JA: Cycling to cancer with cyclin D1. Cancer Biol Ther 1: 226-231, 2002.

40. Shintani M, Okazaki A, Masuda T, Kawada M, Ishizuka M, Doki Y, Weinstein IB and Imoto M: Overexpression of cyclin DI contributes to malignant properties of esophageal tumor cells by increasing VEGF production and decreasing Fas expression. Anticancer Res 22: 639-647, 2002.

41. Yin L, Li H, Liu W, Yao Z, Cheng Z, Zhang H and Zou H: A highly potent CDK4/6 inhibitor was rationally designed to overcome blood brain barrier in gliobastoma therapy. Eur J Med Chem 144: 1-28, 2018.

42. Bajpai R and Nagaraju GP: Specificity protein 1: Its role in colorectal cancer progression and metastasis. Crit Rev Oncol Hematol 113: 1-7, 2017.

43. Guan H, Cai J, Zhang N, Wu J, Yuan J, Li J and Li M: Sp1 is upregulated in human glioma, promotes MMP-2-mediated cell invasion and predicts poor clinical outcome. Int J Cancer 130: 593-601, 2012. 